\title{
A Preliminary Evaluation of Electromyographic Activities of Masseter Muscles during Speech in Myalgia Patients
}

\author{
Eishi Suzuki, DDS, Shoichi Ishigaki, DDS, PhD, Hirofumi Yatani, DDS, PhD, \\ Emiko Morishige, DDS, PhD, and Seiji Omatsu, DDS, PhD \\ Department of Fixed Prosthodontics, Osaka University Graduate School of Dentistry, Osaka, Japan
}

\section{Clinical significance}

Electromyographic activities of masseter muscles during speech were measured without pain in myalgia patients. The mean power frequency during speech was significantly higher in the patient group than the control group, suggesting that speech is a more suitable task than clenching when measuring masseter muscle fatigue in patients with facial pain.

\begin{abstract}
Purpose: To evaluate the electromyographic activities (EMG) of masseter muscles during speech as a method for evaluating muscle fatigue in myalgia patients.

Methods: The control group consisted of 6 subjects (3 males and 3 females, mean age 25.8 yrs), and the patient group consisted of 6 myalgia patients ( 6 females, mean age 29.5 yrs). The bilateral EMG of the masseter muscles were recorded using bipolar surface electrodes and a computer-based EMG analyzer (UAS-108, UNIQUE MEDICAL Co. Ltd.) during the following sessions: rest (30 seconds) / speech / rest (30 seconds) / maximum voluntary contraction (MVC, 5 seconds). Integrated electromyograms (IEMGs) and mean power frequency (MPF) were subjected to statistical comparisons between the two groups with t-test and Pearson's product moment correlation coefficient.
\end{abstract}

Results: No significant difference was found in the IEMG during speech between the two groups, while the myalgia patient group showed significantly lower IEMG than the control group during maximum voluntary contraction (MVC) $(P=.004)$. MPF during speech was significantly higher in myalgia patients $(P=.040)$. The same tendency was observed in MPF during MVC, although the difference was not significant. In the control group, strong correlations were found between MPFs during MVC and speech $(r=.929, P=.007)$, and between IEMGs during MVC and speech $(r=.822$, $P=.045)$. In myalgia patients, no such correlations

Corresponding to: Dr Eishi Suzuki

Department of Fixed Prosthodontics, Osaka University

Graduate School of Dentistry

1-8 Yamadaoka, Suita, Osaka 565-0871, Japan

Tel: +81-6-6879-2946, Fax: +81-6-6879-2947

E-mail: e-suzuki@dent.osaka-u.ac.jp

Received on November 15, 2006 / Accepted on May 2, 2007 were observed.

Conclusion: The results from this study may suggest the possibility that MPF during speech can be a method for evaluating masticatory muscle fatigue.

Key words: myalgia, speech, masseter muscle, mean power frequency, EMG

\section{Introduction}

Chewing, ${ }^{1,2}$ clenching $^{3-12}$ and tapping ${ }^{13,14}$ movements are usually performed when masticatory muscle function is electromyographically evaluated. However, since these tasks are likely to cause pain in myalgia patients, and the occlusal condition can affect the electromyographic activities (EMG), ${ }^{8}$ these tasks are not necessarily suitable for measuring masticatory muscle fatigue. Our final goal is to develop a method for judging therapeutic gain by objectively evaluating masticatory muscle fatigue with no pain and no influence of the occlusal condition. To reach this goal, we are studying if the speech movement can be an alternative test movement to chewing, clenching or tapping. In this pilot study we evaluated the EMG activities of masseter muscles during speech in myalgia patients and compared the activities with those in normal subjects.

\section{Materials and methods}

The control group consisted of 6 subjects ( 3 males and 3 females, mean age $25.8 \mathrm{yrs}$ ), and the patient group consisted of 6 myalgia patients ( 6 females, mean age 29.5 yrs). Pain on palpation of the masseter muscle was evaluated on a point scale of 0-3; 0 (no pain), 1 (tender or sore feeling), 2 (clear pain), and 3 (severe pain with escape response). There was no significant difference in the age between the two groups $(P=.264)$. Of patients with total scores of $0-6$, patients with 
scores of 4 or more were defined as having myalgia. This study was approved by the ethical committee of Osaka University Graduate School of Dentistry and informed consent was obtained from all subjects and patients prior to entering the study.

A short Japanese sentence (Niwa No Ishigaki Ni Sotte Iki-iki To Saku Kiku No Hana No Mae De Shibashi Tachidomarareta) was used as the test sentence. This is one of the test sentences that our department developed for the kinesiological assessment of jaw movement. ${ }^{15,16}$ Since it has been reported that muscle activity was positively correlated with speech rate and intensity, ${ }^{17}$ the reproducibility of muscle activities during speech of this test sentence was preliminarily evaluated prior to starting the present study using 8 healthy volunteers ( 7 males, 1 female). The EMGs during the speech of this test sentence were recorded on two non-consecutive days. The reproducibility of the mean power frequency (MPF) and integrated electromyograms (IEMGs) findings (i.e. intraclass correlation coefficients, ICC) were .674 and .680 , respectively.

The bilateral EMG of masseter muscles were recorded using bipolar surface electrodes (DELSYS $^{\circledR}$, DELSYS Inc.) and a computer-based EMG analyzer (UAS-108, UNIQUE MEDICAL Co. Ltd.) during a series of experimental sessions: rest (30 s) / speech / rest (30 s) / maximum voluntary contraction (MVC, $5 \mathrm{~s}$ ). This sequence was determined based on a preliminary test to shorten the experiment time and not to induce muscle fatigue during the experiment. It was confirmed that the EMG activities during speech returned to the EMG activities of the baseline within $30 \mathrm{~s}$, while the EMG activities during MVC did not return to the EMG activities of the baseline within $30 \mathrm{~s}$. The EMG signals were sampled at $1,000 \mathrm{~Hz}$ and filtered with band pass between $10 \mathrm{~Hz}$ and $490 \mathrm{~Hz}$. Each subject was seated on a chair with the head unsupported and relaxed. The test sentence was set up in front of a subject so that the subject could read it. The surface electrodes were cleaned with $80 \%$ alcohol swabs and placed on the center of the belly of bilateral masseter muscles. The electrode housing was constructed with a waterproof polyurethane plastic case, which was internally shielded to reject ambient electrical noise. The electrode contacts were made from $99.9 \%$ pure silver bars measuring $10 \mathrm{~mm}$ in length, $1 \mathrm{~mm}$ in diameter and spaced $10 \mathrm{~mm}$ apart for optimal signal detection. The ground electrode was attached to an earlobe.

For speech movement, the entire duration of data during speech was analyzed. For MVC, an arbitrary $1 \mathrm{~s}$ of the $5 \mathrm{~s}$ recording was analyzed. We used MPF $(\mathrm{Hz})^{4-8,10,11}$ for qualitative analysis and IEMG $(\mu \mathrm{V} \cdot \mathrm{s})^{1,8}$ for quantitative analysis. The EMG signals were rectified and integrated. The IEMG was converted into common logarithm to facilitate comparison of data in sight. Since no significant difference was found in MPF and IEMG between left and right sides, the mean value of MPF and IEMG on both sides was subjected to analysis. In the frequency domain analysis, 2048-point running FFTs (Fast Fourier Transforms) was used with a Hanning window. IEMG and MPF were statistically compared between the two groups with t-test and Pearson's product moment correlation coefficient using SPSS $^{\circledR}$ 14.0J. The level of significance was set at $\alpha=.05$.

\section{Results}

The mean time required for speech was $5.1 \mathrm{~s}$ in the healthy control group and $6.2 \mathrm{~s}$ in the myalgia patient group. There was no significant difference in the time between the two groups $(P=.114)$. No significant difference was found in IEMG during speech between the two groups $(P=.835$, Fig. 1), while the myalgia patient group showed significantly lower IEMG than the control group during MVC ( $P=.004$, Fig. 1). MPF during speech was significantly higher in myalgia patients $(P=.040$, Fig. 2). The same tendency was also observed in MPF during MVC, but the difference was not significant ( $P=.068$, Fig. 2$)$. In the control group, strong positive correlations were found between MPFs during MVC and speech $(r=.929$, $P=.007$, Fig. 3), and between IEMGs during MVC and speech ( $r=.822, P=.045$, Fig. 4$)$. On the other hand, no such correlations were observed in the myalgia patients (MPF: $r=.680, P=.137$, Fig. 3, and IEMG: $r=-.210, P=.690$, Fig. 4$)$.

\section{Discussion}

\section{MPF during MVC}

It has been reported that MPF during MVC shifted to lower frequencies ${ }^{4-8}$ and this phenomenon has been considered to represent muscle fatigue. Since most myalgia patients in this study report- 


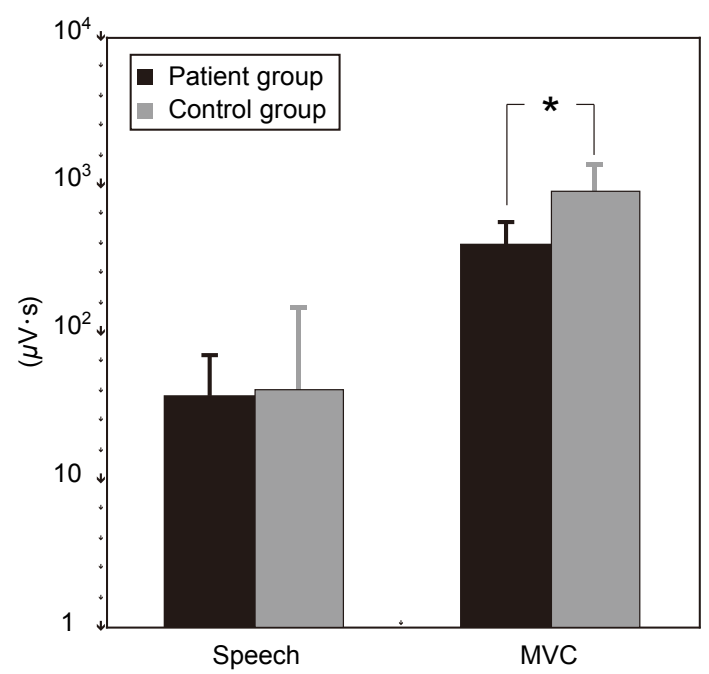

Fig. 1 IEMG during speech and MVC in myalgia patient group and control group $(*: P=.004)$.

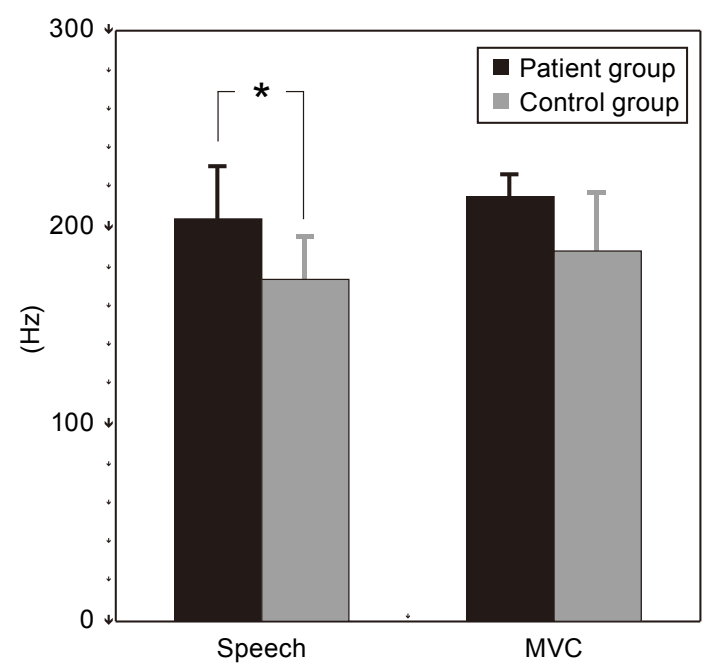

Fig. $2 \mathrm{MPF}$ during speech and MVC in myalgia patient group and control group (*: $P=.040)$.

ed muscle fatigue or pain, it was expected that MPF would be significantly lower in the myalgia patient group than in the control group during MVC. However, the results of this study revealed that MPF during MVC was slightly higher in the myalgia patient group than in the control group, although the difference was not significant. The bite force during MVC has been reported to be lower in the TMD patients than in the control groups. ${ }^{3,5,8,9}$ In addition, with increasing bite force magnitude, a significant negative trend of MPF has also been reported for the masseter. ${ }^{5}$ In the present study, although the bite force was not recorded, we expected that myalgia patients would not be able to bite at maximum force due

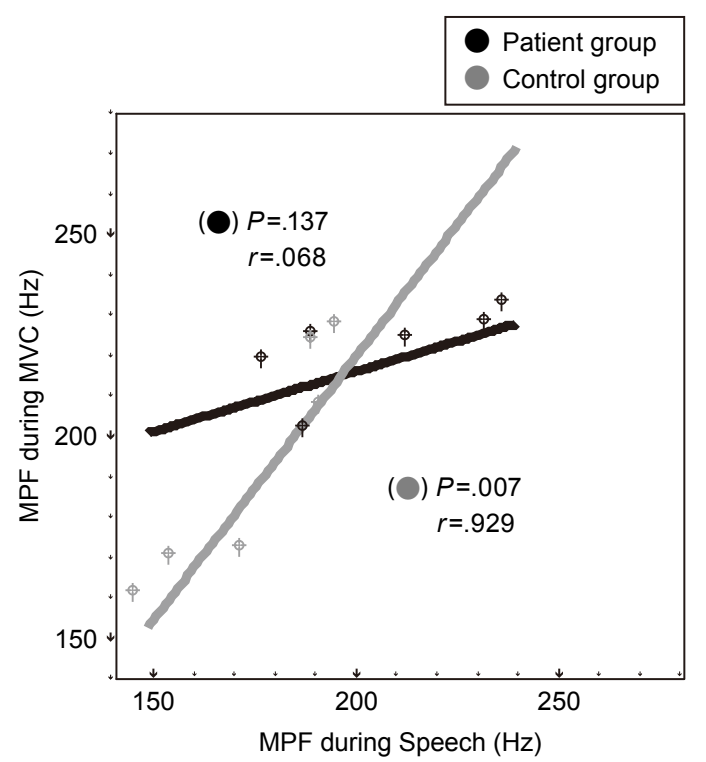

Fig. 3 Correlation between MPFs during speech and MVC in myalgia patient group and control group.

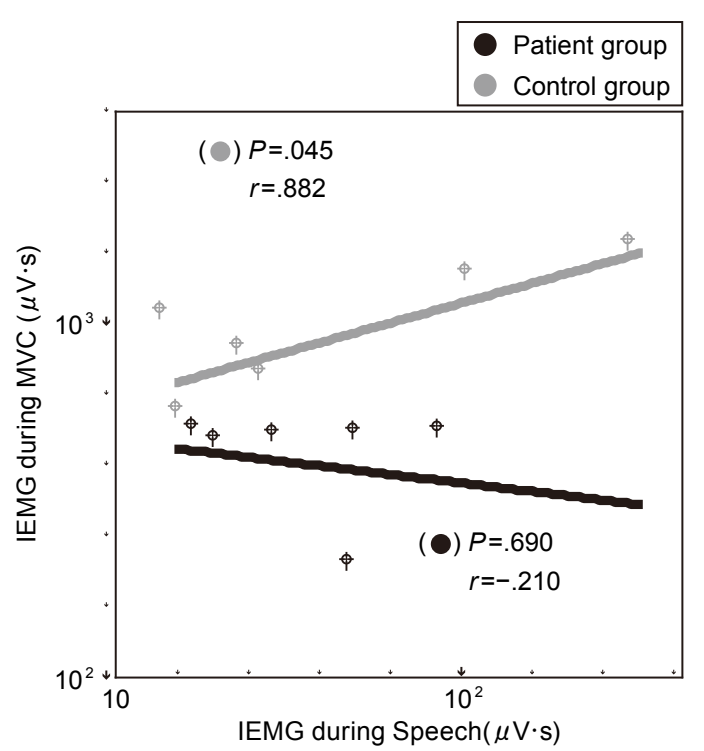

Fig. 4 Correlation between IEMGs during speech and MVC in myalgia patient group and control group.

to muscle pain. In fact, the IEMG during MVC was significantly lower in the myalgia patient group than in the control group. The significant difference of IEMG between the two groups could result in lower bite force, and hence, a slightly higher MPF in the myalgia patient group.

\section{MPF during speech}

The differences in MPF during speech between the myalgia patient group and the healthy control group have never been reported. In the pres- 
ent study, the myalgia patient group showed significantly higher MPF than the control group, although there was no significant difference in IEMG between the two groups. No subjects felt pain during speech, which was entirely different than when it was during MVC. This may explain why no significant difference was found in IEMG during speech between the two groups. Although it is difficult to determine the reasons for higher MPF during speech in myalgia patients, it could be supposed that in myalgia patients some changes in the recruitment pattern or rate coding of motor units may have taken place during speech, probably due to muscle fatigue or a dysfunction of the masseter. In myalgia patients, masseter muscles could be under fatigue because of nocturnal or diurnal clenching. ${ }^{4,12}$ Since these parafunctional activities almost does not come up to a little less than $50 \% \mathrm{MVC},{ }^{4,12,18}$ the type 1 fibers and the type 2 fibers are not all recruited at the same time, rather, the type 1 fibers are mainly recruited. ${ }^{5}$ It could be supposed that in myalgia patients the number of firing type 1 fibers decreased because of fatigue, and were covered instead by more type 2 fibers being recruited or increased firing rate of the type 2 fibers. In fact, Wretling et al $(1987)^{19}$ showed that as the percentage of type 1 fibers decreased, MPF increased in the vastus lateralis muscle. The change of the firing fiber type proportion might explain why MPF during speech was significantly higher in myalgia patients. These results showed that MPF during speech was higher in the myalgia patient group than in the control group, although there was no significant difference in IEMG between the two groups, which also suggests that speech may therefore be a potentially effective and lowinvasive test movement for measuring the muscle fatigue in myalgia patients.

\section{Correlation between MVC test and speech test}

In the control group, a significant positive correlation was found between MPFs during MVC and speech (Fig. 3), and a similar correlation was also found between IEMGs during MVC and speech (Fig. 4). These results show that, in the control group, the higher IEMG and MPF during MVC are associated with the higher IEMG and MPF during speech. It is thus suggested that, in healthy subjects, similar results can be obtained both in MVC and speech. In other words, speech movement is as appropriate as MVC in assess- ing the EMG activities of masseter muscles in healthy subjects. On the other hand, no such correlation was found in the myalgia patient group. The results mean that patients who show high IEMG and MPF during MVC do not always show high IEMG and MPF during speech in myalgia patients. In figures 3 and 4, it can be recognized that both MPF and IEMG during MVC distribute within a small range in the patient group. The finding can probably be explained by the weak occlusal force during MVC in most myalgia patients. On the contrary, MPF and IEMG during speech distribute in a broader range and show significant variations among subjects both in the patient and control groups. These findings show the possibility that, as a test movement, speech might reflect individual muscle function more specifically than MVC. In addition, speech movement is not likely to produce pain during mandibular movements. Therefore, speech movement may be more appropriate than MVC when electromyographically assessing the muscle fatigue, especially in myalgia patients.

In the present study, the sex ratio was not matched and the number of samples is small. A further study investigating age- and sexmatched groups with a greater sample size is going to be reported in the near future.

\section{Conclusion}

While there was no significant difference in MPF during MVC between myalgia patients and control subjects, MPF during speech was significantly higher in myalgia patients. The result may suggest the possibility that masticatory muscle fatigue can be objectively measured by MPF during speech. Further study is needed to prove this possibility.

Acknowledgments: This study was partially supported by a Grant-in-Aid for Scientific Research (C17592020) from the Japan Society for the Promotion of Science.

\section{References}

1. Sato S, Kawamura H. Natural course of nonreducing disc displacement of the temporomandibular joint: changes in electromyographic activity during chewing movement. J Oral Rehabil 32: 159-165, 2005. 
2. Soboleva U, Laurina L, Slaidina A. The masticatory system - an overview. Stomatologija 7: 77-80, 2005.

3. Tortopidis D, Lyons MF, Baxendale RH. Bite force, endurance and masseter muscle fatigue in healthy edentulous subjects and those with TMD. J Oral Rehabil 26: 321-328, 1996.

4. Svensson P, Burgaard A, Schlosser S. Fatigue and pain in human jaw muscles during a sustained, low-intensity clenching task. Arch Oral Biol 46: 773-777, 2001.

5. Farella M, Eijden TV, Baccini M et al. Task-related electromyographic spectral changes in human masseter and temporalis muscles. Eur J Oral Sci 110: 8-12, 2002.

6. Hori H, Kobayashi H, Hayashi T et al. Mean power frequency shift during fatigue and recovery in patients with craniomandibular disorders. J Oral Rehabil 22: 159-165, 1995.

7. Gay T, Maton B, Rendell J et al. Characteristics of muscle fatigue in patients with myofascial paindysfunction syndrome. Arch Oral Biol 39: 847-852, 1994.

8. Liu ZJ, Yamagata K, Kasahara Y et al. Electromyographic examination of jaw muscles in relation to symptoms and occlusion of patients with temporomandibular joint disorders. J Oral Rehabil 26: 33-47, 1999.

9. Wang K, Arima T, Arendt-Nielsen L et al. Emgforce relationships are influenced by experimental jaw-muscle pain. J Oral Rehabil 27: 394-402, 2000.

10. Yuen SW, Hwang JC, Poon PW. EMG power spectrum patterns of anterior temporal and masseter muscles in children and adults. J Dent Res 68: 800-804, 1989.
11. Westbury JR, Shaughnessy TG. Associations between spectral representation of the surface electromyogram and fiber type distribution and size in human masseter muscle. Electromyogr Clin Neurophysiol 27: 427-435, 1987.

12. Glaros AG, Burton E. Parafunctional clenching, pain, and effort in temporomandibular disorders. J Behav Med 27: 91-100, 2004.

13. Saifuddin MD, Miyamoto K, Ueda HM et al. A quantitative electromyographic analysis of masticatory muscle activity in usual daily life. Oral Dis 7: 94-100, 2001.

14. Munakata Y, Tsuji M, Kasai S. Occlusal force pattern during rhythmic human tapping movement. J Oral Rehabil 18: 265-272, 1991.

15. Yoshioka C, Ogawa H, Kuwahara T et al. A prosthetic study on mandibular movements during speech. Part 1. The mandibular position during phonation of the Japanese syllabary. J Jpn Prosthodont Soc 36: 817-822, 1992.

16. Ogawa H, Yoshioka C, Sanma S et al. Consistency of mandibular movements during speech. J Osaka Dent Univ 37: 539-546, 1992 (in Japanese).

17. McClean MD, Tasko SM. Association of orofacial muscle activity and movement during changes in speech rate and intensity. J Speech Lang Hear Res 46: 1387-1400, 2003.

18. Piquero K, Sakurai K. A clinical diagnosis of diurnal (non-sleep) bruxism in denture wearers. J Oral Rehabil 27: 473-482, 2000.

19. Wretling ML, Gerdle B, Henriksson-larsen K. EMG: a non-invasive method for determination of fiber type proportion. Acta Physiol Scand 131: 627-628, 1987. 